\title{
Whiskers growth and self-healing in Ti-based metallic glasses during ion irradiation
}

\author{
Kun Zhang ${ }^{\mathrm{a}, \mathrm{b}}$, Zheng $\mathrm{Hu}^{\mathrm{c}}$, Ziqiang Zhao ${ }^{\mathrm{d}}$, Bingchen Wei ${ }^{\mathrm{a}, \mathrm{b}, *}$, Yansen $\mathrm{Li}^{\mathrm{a}}{ }^{\mathrm{a} b}$, Yuhang Wei ${ }^{\mathrm{a}, \mathrm{b}}$ \\ ${ }^{a}$ Key Laboratory of Microgravity (National Microgravity Laboratory), Institute of Mechanics, Chinese Academy of Sciences, Beijing 100190, China \\ b School of Engineering Science, University of Chinese Academy of Sciences, Beijing 101408, China \\ ' Science and Technology on Vehicle Transmission Laboratory, China North Vehicle Research Institute, Beijing 100072, China \\ d School of Physics, Peking University, Beijing 100871, China
}

\section{A R T I C L E I N F O}

\section{Article history:}

Received 24 July 2017

Received in revised form 19 October 2017

Accepted 18 December 2017

Available online 21 December 2017

\section{Keywords:}

Metallic glasses

Ion irradiation

Stress distribution

Whiskers growth

Self-healing

\begin{abstract}
A B S T R A C T
Ti-based metallic glasses were subjected to a $20 \mathrm{MeV} \mathrm{Cl}^{4+}$ ion radiation under liquid-nitrogen cooling. Their responses, as well as effects of the electronic excitation and nucleus-nucleus collision were evaluated. The collision cascade during irradiation typically changes the structure by increasing the liquid-like zone/cluster, or the content of the free volume. However, along the ion incident depth, the structure change is inhomogeneous. Numerous whiskers appear and aggregate on the side of the irradiation surface, which are several micrometers away from the edge. This corresponds with the maximum collision depth obtained by the Monte Carlo simulation, where nuclear loss plays a dominant role. Moreover, the liquid-like zone continually forms, which add to the whiskers growth and subsequent self-healing. Results suggest that the irradiation-induced local shear stress combines with the well-localized liquidlike zone results in the observed phenomena. This study demonstrates that metallic glasses have high morphological instability under ion irradiation, which assets can pave new paths for their further applications.
\end{abstract}

(c) 2017 Elsevier B.V. All rights reserved.

\section{Introduction}

Metallic glasses (MGs) have been a popular subject of research within the past decade, due to their unique mechanical and chemical properties, including high specific strength and good corrosion resistance [1-5]. They also exhibit inherent disordered structures, making them ideal candidates for applications in nuclear-irradiated environments or deep space. Many studies focus on the intrinsic irradiation responses by MGs, including damage morphology [6], enhanced mechanical performance [7], and local structure change $[8,9]$. Studies have shown that the ion beam resistance capability of MGs is better than either their corresponding crystalline counterparts or traditional radiation-resistant materials [10,11]. The entirely disordered structure is considered a desirable environment to relax the damage induced by radiation as well as local shear stress. The random nature as "frozen liquids", where the local liquid regions along the thermal spike can rearrange themselves and

\footnotetext{
* Corresponding author.

E-mail address: weibc@imech.ac.cn (B. Wei).
}

solidify without strong boundary constraints from the surrounding crystalline grain [12].

The anisotropic deformation is a unique phenomenon that occurs in amorphous thin films (such as in a-Si and MG films) during hundreds of MeV ion irradiation [13]. Previous studies have shown that the high electronic energy deposition and low directional straggle in the ion trajectory causes a cylindrically shaped narrow thermal spike to evolve around the ion track. Plastic relaxation of the local shear stress induced the by thermal expansion of this hot cylindrical region, can result in global plastic deformation perpendicular to the cylindrical axis. Therefore, the amorphous thin film tends to undergo a homogeneous anisotropic growth where the incident ions act like a hammer [14].

The two basic aspects that induce the thermal spike effect are the electronic excitation and nucleus-nucleus collision. Both types of spikes could be important within $\mathrm{MeV}$ range heavy ions, contingent on the magnitude between the electronic loss and the nuclear loss. These resulting thermal spikes differ in that electronic excitations have cylindrical geometry that completely breaks the isotropic symmetry of the system, whereas nucleus-nucleus collisions have near spherical geometry, where the plastic deformation is localized in the maximal collisional damage region [15]. 
However, few studies have focused on the nucleus-nucleus collision effects on the anisotropic growth of MGs through changes in the incident ion energy.

Experimental observations have shown that the MGs are intrinsically structurally inhomogeneous. The MGs could be viewed as tightly bonded atomic clusters that overlap and form the percolating "skeleton" of the glassy structure, with loosely bonded free-volume regions acting as liquid-like defects when subjected to an external force [16]. Bian et al. suggested that irradiation at low dosages could alter the structure by increasing the freevolume fraction, namely the liquid-like zone [17]. Petrusenko et al. reported the occurrence of $\mathrm{MeV}$ electron irradiation damage, suggesting the existence of vacancy-type defects and interstitial-type defects found in the MGs correspond to density fluctuations [18]. The microstructure evolution, as well as correlation between the structure heterogeneity and anisotropic growth, should be investigated to further clarify the influence of irradiation on MGs.

The following study investigates the responses of Ti-based MGs made by the $\mathrm{Cl}^{4+}$ ion irradiation under liquid-nitrogen cooling. $\mathrm{Ti}_{40} \mathrm{Zr}_{25} \mathrm{Be}_{30} \mathrm{Cr}_{5}$ MGs have high GFA, exceptional thermal stability, low density (comparable to that of pure $\mathrm{Ti}$ ) and very high specific strength, which may be ideal candidates for applications in deep space. The incident ion energy is $20 \mathrm{MeV}$, which much smaller than the energy reported that induced homogeneous anisotropic deformation of the MGs within hundreds of MeV ion irradiation [13]. Whiskers growth is found on the side of MGs during irradiation. More importantly, the self-healing phenomenon of the MG ribbons with microcosmic flaw is observed. The kinetics and related mechanism for whiskers formation and self-healing in MGs are discussed.

\section{Experimental}

$\mathrm{Ti}_{40} \mathrm{Zr}_{25} \mathrm{Be}_{30} \mathrm{Cr}_{5}$ (Ti-based) MGs with a $5 \mathrm{~mm}$ diameter were prepared by arc melting a mixture of elements with purities greater than $99.9 \%$ in an argon atmosphere, and then chilled casted into a copper mold. Ti-based MG ribbons $(2.0 \times 0.02 \mathrm{~mm}$ cross sections) were obtained at a rotation speed of $40 \mathrm{~m} / \mathrm{s}$ via melt spinning. The surfaces of the samples were mechanically polished to a mirror finish and cleaned ultrasonically prior to the irradiation experiments. After that, the irradiation experiments were completed on the $2 \times 6 \mathrm{MV}$ EN tandem accelerator platform at Peking University. The prepared samples were irradiated in a normal incidence for $2.4 \mathrm{~h}$ at a dosage rate of $1.6 \times 10^{14}$ ions $\mathrm{cm}^{-2} \mathrm{~s}^{-1}$. During the entire irradiation process, the samples were cooled by liquid nitrogen. The X-ray diffraction (XRD) analysis was carried out on a Philips PW1050 diffractometer using $\mathrm{CuK} \alpha$ radiation. The microstructural morphology was tested by scanning electron microscope (SEMEDS, JEM-2100F).

The parameters of the ion beams and displacement damage were calculated using SRIM code [19]. The number of defects per ion $N$ and the ion penetration depth $d$ are provided by the SRIM calculations. Then, the damage level $d p a$ is calculated as follows [20]:

$d p a=\frac{F N M_{m o l}}{\rho \mathrm{d} N_{A}}$

F is the ion fluence, $M_{m o l}$ the target's molar mass and $N_{A}$ Avogadro's constant.

\section{Results and discussion}

The surface morphology of Ti-based MGs irradiated by different doses is shown in Fig. 1. The Ti-based MG retained its original state in a low ion irradiation dose $\left(\phi=5.15 \times 10^{15}\right.$ ions $\left.\mathrm{cm}^{-2}\right)$, with no significant changes observed on the surface of the Ti-based MGs. When the irradiation exceeded a critical dose $\left(5.15 \times 10^{15}\right.$ ions $\mathrm{cm}^{-2}<\phi<6.44 \times 10^{15}$ ions $\mathrm{cm}^{-2}$ ), numerous whiskers appeared and aggregated on the unirradiated edge. As shown in Fig. 1e, the whiskers grow on the unirradiated edge, but not directly on it. In fact, these whiskers are several micrometers away from the edge of the irradiation surface. As the irradiation dose increases, whiskers become bent and closely interwoven. The relation between the average whiskers length and irradiation dose is shown in Fig. 2. The rate for whiskers growth remains constant (about $1.17 \mu \mathrm{m} / \mathrm{dpa}$ ) without saturation until the maximum irradiation dose is reached.

Whiskers growth has been widely reported in other materials, such as, Sn or Zn crystal solders [21,22]. Whiskers growth in Sn solders can be interpreted as an atomic diffusion process where stress generation and relaxation simultaneously occurs. To determine the origin of whiskers growth in MGs, the XRD and the EDS analysis are used to establish the phase and chemical composition of the whiskers and the substrates. Both the irradiated and as-cast Ti-based MGs exhibit a single broad diffraction peak in the XRD curves, confirming an amorphous structure at near-surface (Fig. 3). The peak intensity of the irradiated sample exhibits a slight drop when compared with the as-cast sample, demonstrating that the amorphous state become more disordered as the accelerated $\mathrm{Cl}^{4+}$ ion sets into the matrix. The EDS results show the chemical composition of the whiskers are similar to the one of matrix, which suggests that the diffusion mechanism for whiskers growth may be not an appropriate mechanism in determining whiskers appearance in MGs.

The surface-confined viscous flow has been proven to play the dominant quantitative role during ion irradiation. This is caused by radiation-induced softening of the near-surface layer $[11,23]$. The origin of the softened region is caused by the rearrangements of atoms in certain structurally weak spots that behave as the liquid-like zone or the free volume. However, this does not explain why whiskers grow on the unirradiated edge. Further research that addresses the structural response, stress distribution, and the nature of whiskers should be carried out.

The electronic excitation and nucleus-nucleus collision are two features involved in the anisotropic growth induced by the thermal spike effects. These two concepts also account for most of the observed property changes occurring from atomic displacement by high-energy ions in MGs. Fig. 4 shows the change of energy loss, as well as the collisional damage distribution along the ion incident direction. There are three typical regions can be divided base on the incident ion track: the electronic loss-dominated region (Region I), the nuclear loss-dominated region (Region II) and the unaffected region (Region III) (see Fig. 4a). These regions can also be referred to as the defect-starting region, the defect-rich region, and the matrix region seen in Fig. 4b. In the electronic loss-dominated region, the cross section affected by irradiation is small with minimal damages occurring because of the initial high incident energy. Nuclear loss plays a dominant role as the ions penetrate into deeper regions. As shown in Fig. 4, the incident ion results in the maximum collisional damage occurring at a depth of $5 \mu \mathrm{m}$, this corresponds to the location of whiskers initiation. Therefore, we believe that the nucleus-nucleus collision played a dominant role on whiskers anisotropic growth within this study.

In order to clarify the origin of whiskers growth in MGs, the schematic diagram of viscosity variation and structure evolution is shown in Fig. 5. The MGs exhibit the largest atomic rearrangement and the least amount of viscosity at the maximum collisional damage depth. Moreover, the irradiation response is inhomogeneous along the ion incident direction. The defect-rich region sustains a larger increase of the liquid-like region than the defect-starting region, as seen in Fig. 5. The increase of liquid-like region indicates that the atoms in the defect-rich region are more loosely packed than those in the defect-starting region. If we assumed a uniform 


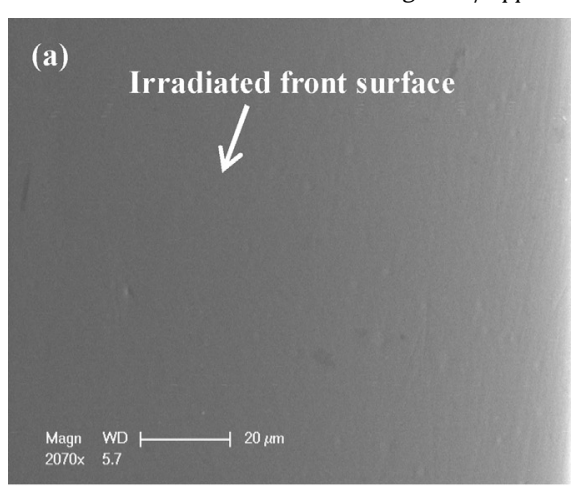

(b)
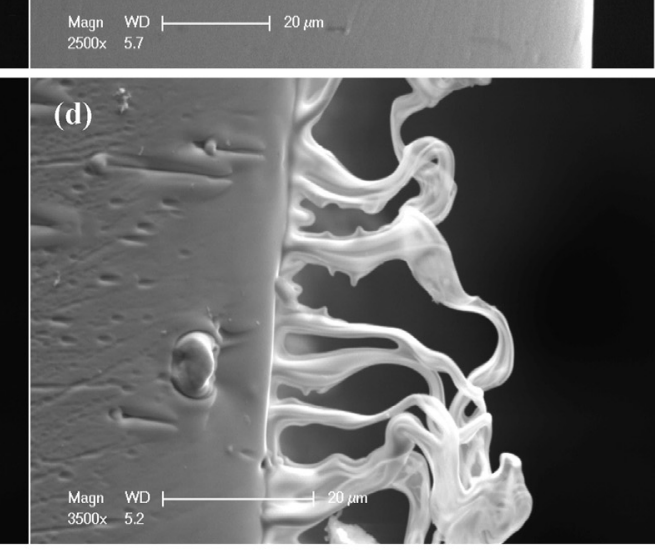

(e)

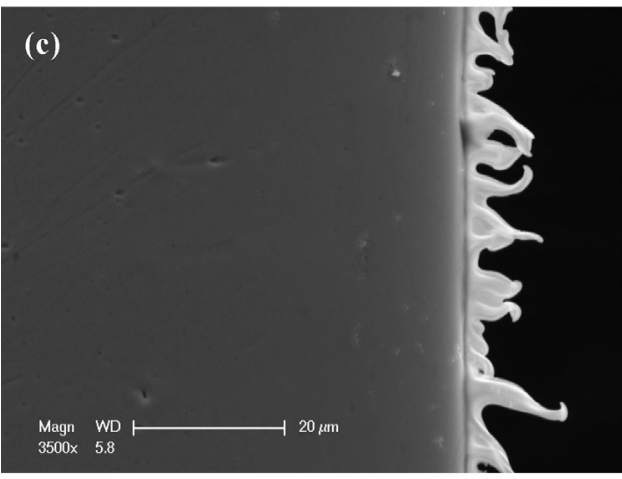

Irradiated front surface

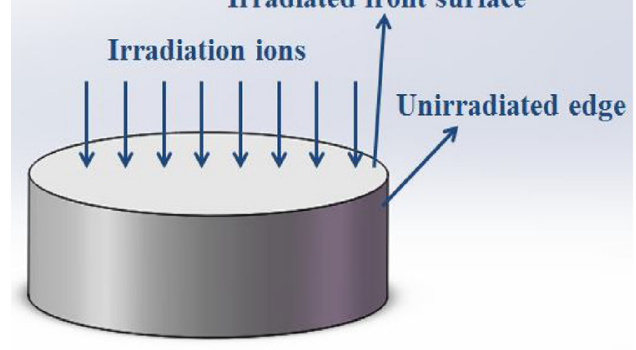

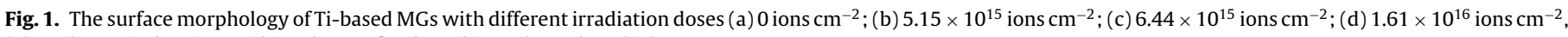
(e) a schematic drawing with ion beams for describing where the whisker grow out.

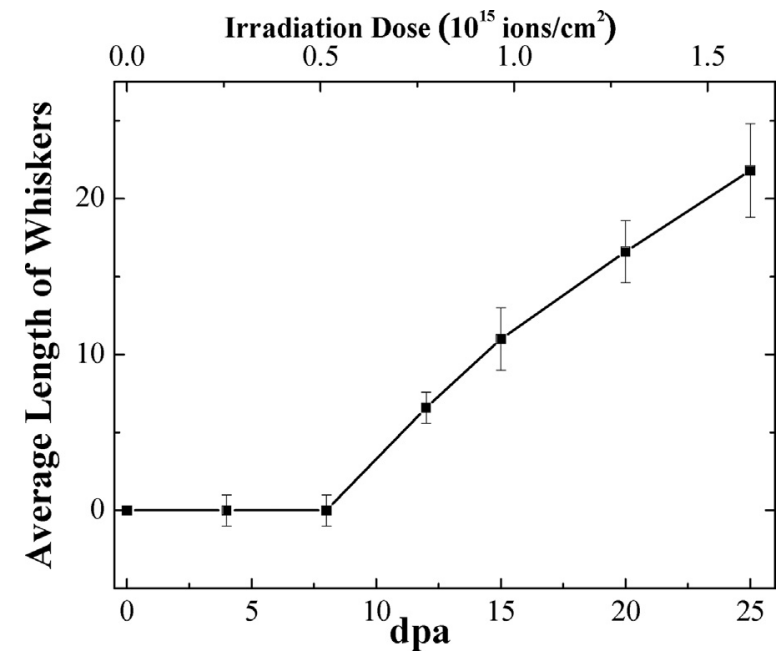

Fig. 2. The average whiskers length as related to the increase of the irradiation dose or dpa. temperature distribution in the thermal spike region, the stresses inside the ion track region [24] are obtained by the following:

$\sigma_{r r}=\sigma_{\theta \theta}=\frac{1}{2} \sigma_{z z}=-\frac{\alpha E \Delta T}{2(1-v)}\left(1-\delta^{2}\right)$

where $E$ is Young's modulus, $v$ is Poisson's ratio, and $\delta=\mathrm{a} / \mathrm{b}, \Delta T$ is the temperature difference between the thermal spike affected and unaffected region. This suggests a local shear stress occurs perpendicular to the ion incident direction. The defect-rich region may accommodate larger shear stress than the defect-start region because it has a more severe volume expansion. Those weak spots may preferentially travel towards the local low-stress regions in order to unload the stress. The liquid-like zone aggregates, moving toward to the side of the MGs. This is a possible factor in whiskers generation. Actually, the irradiation-induced stress and structural relaxation have been studied by Volkert [25]. A larger expansion $(0.3 \%)$ is observed on heating liquid-nitrogen-temperature irradiated amorphous layer to room temperature. The behavior reveals the irradiation at liquid-nitrogen temperature can accumulate larger stress and more defects owing to the suppression of atomic mobility, which would result in whiskers growth on the unirradiated edge.

Once whiskers appear, the liquid-like zone forms continually, supplying resources to the whiskers until the local stress is relaxed. 


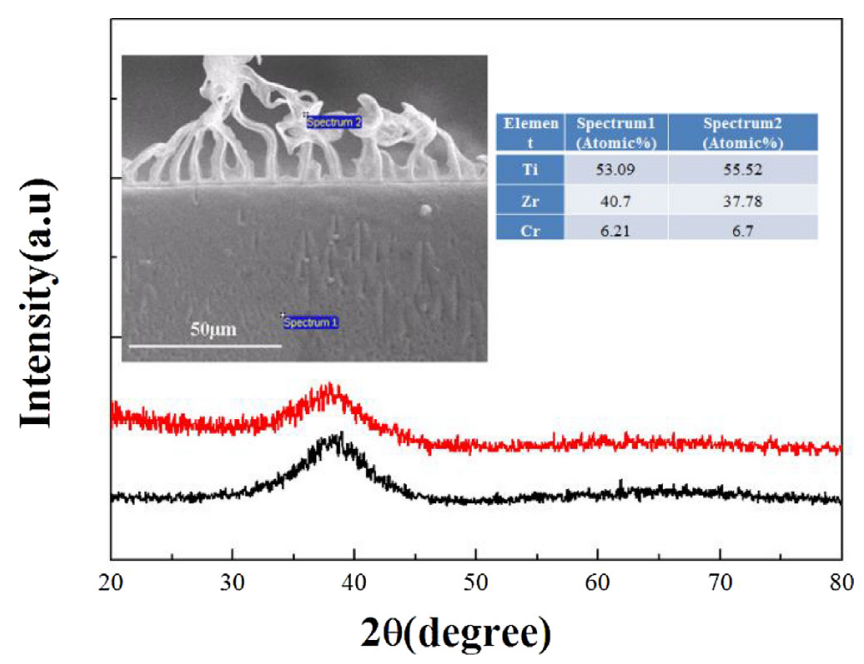

Fig. 3. The XRD results of the as-cast (Black) and irradiated samples (Red). Insert: the chemical composition analysis of the whiskers and matrix after irradiation. (For interpretation of the references to color in this figure legend, the reader is referred to the web version of this article.)

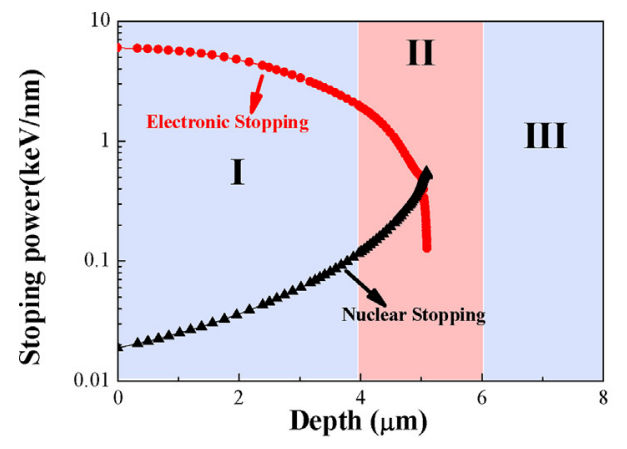

Fig. 6 shows the irradiation response of Ti-based MG ribbons that possess many microcosmic flaws in center regions. It is noted that the microcosmic flaws eventually disappeared during irradiation, due to whiskers growth on the unirradiated edge. We suggest that the irradiation-induced stress combines with well-localized liquidlike zone to cause whiskers growth and self-healing in the MGs. Ion irradiation may act as an effective method for designing micronano structures or repairing micro-crack and microcosmic flaws by controlling the whiskers growth in MGs.

\section{Conclusion}

In this study, Ti-based MGs are subjected to a $20 \mathrm{MeV} \mathrm{Cl}{ }^{4+}$ ion radiation under liquid-nitrogen cooling and their responses are observed. Numerous amorphous whiskers appear and aggregate near the irradiated surface, corresponding to the maximum collision defect. The liquid-like zone continually forms to enable whiskers growth and self-healing as the irradiation dose increases. The irradiation-induced stress, combined with well-localized liquid-like zone, is believed to cause this whiskers formation as well as self-healing.

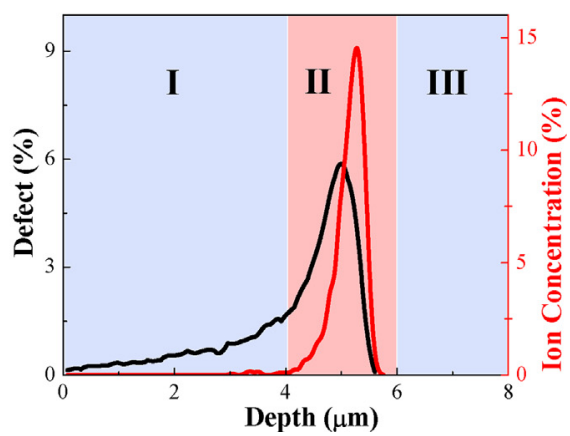

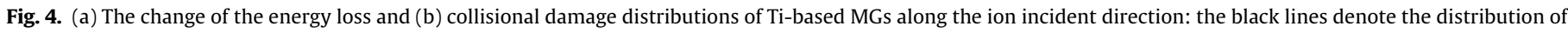

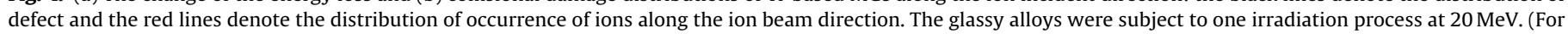
interpretation of the references to color in this figure legend, the reader is referred to the web version of this article.)

(a)
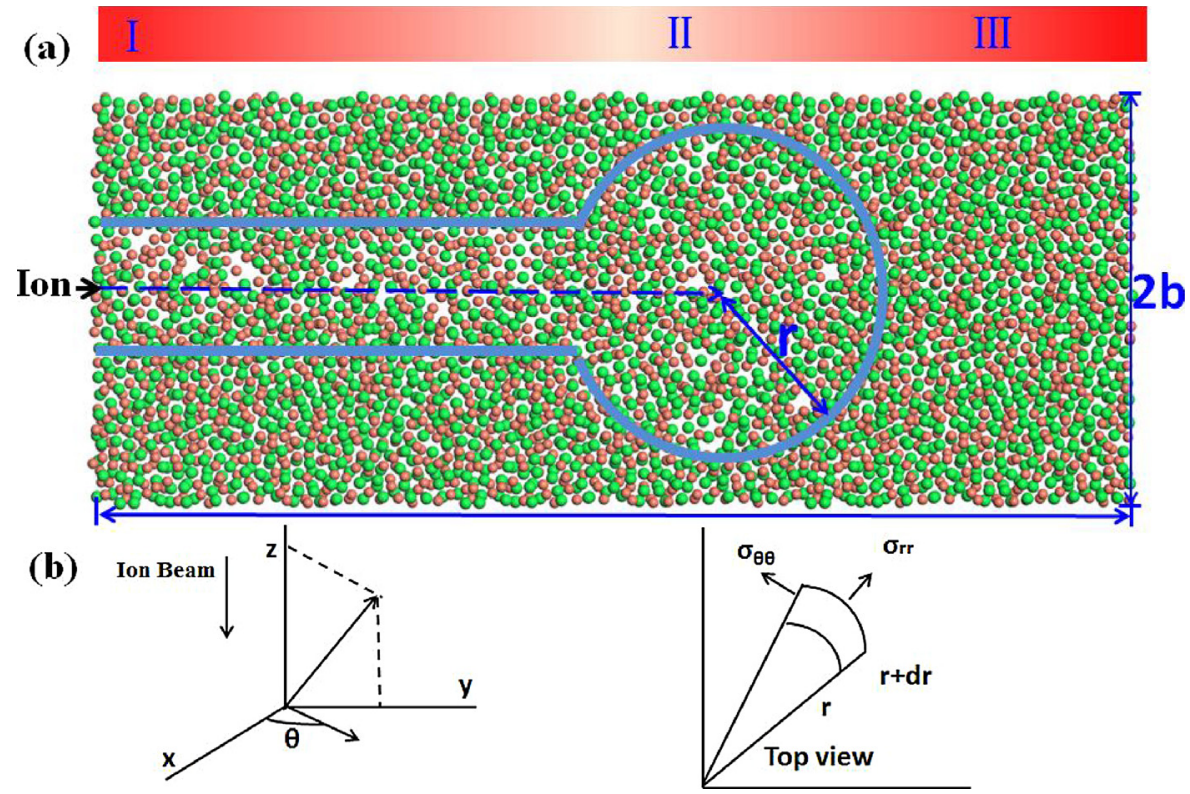

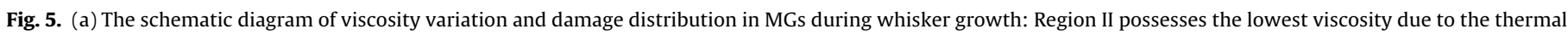
spike effect; (b) the stress analysis in the region affected by the thermal spike. 


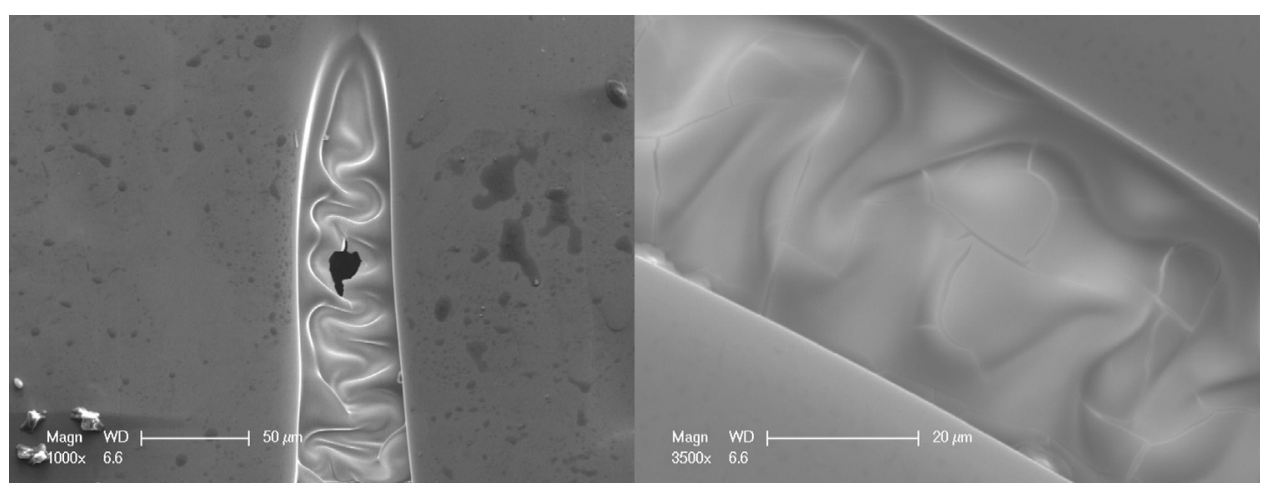

Fig. 6. Self-healing phenomenon of MG ribbons due to whiskers growth.

\section{Acknowledgments}

The authors would like to acknowledge the support by the National Natural Science Foundation of China (Grant No. 51401028, No. 51271193, No. 11402277) and the Strategic Priority Research Program of the Chinese Academy of Sciences (Grant No. XDB22040303). The authors also thank to the support of Opening Fund of State Key Lab of Nuclear Physics and Technology at Peking University.

\section{References}

[1] A.L. Greer, E. Ma, Bulk metallic glasses: at the cutting edge of metals research, MRS Bull. 32 (2011) 611-619.

[2] K.K. Song, S. Pauly, Y. Zhang, S. Scudino, P. Gargarella, K.B. Surreddi, U. Kühn, J. Eckert, Significant tensile ductility induced by cold rolling in $\mathrm{Cu}_{47.5} \mathrm{Zr}_{47.5} \mathrm{Al}_{5}$ bulk metallic glass, Intermetallics 19 (2011) 1394-1398.

[3] W.H. Wang, R.J. Wang, F.Y. Li, D.Q. Zhao, M.X. Pan, Elastic constants and their pressure dependence of $\mathrm{Zr}_{41} \mathrm{Ti}_{14} \mathrm{Cu}_{12.5} \mathrm{Ni}_{9} \mathrm{Be}_{22.5} \mathrm{C}_{1}$ bulk metallic glass, Appl. Phys. Lett. 74 (1999) 1803-1805.

[4] J. Das, M.B. Tang, K.B. Kim, R. Theissmann, F. Baier, W.H. Wang, J. Eckert, "Work-hardenable" ductile bulk metallic glass, Phys. Rev. Lett. 94 (2005) 205501.

[5] W.H. Wang, C. Dong, C.H. Shek, Bulk metallic glasses, Mater. Sci. Eng. R 44 (2004) 45-89.

[6] M.D. Rodríguez, B. Afra, C. Trautmann, M. Toulemonde, T. Bierschenk, J. Leslie, R. Giulian, N. Kirby, P. Kluth, Morphology of swift heavy ion tracks in metallic glasses, J. Non-Cryst. Solids 358 (2012) 571-576.

[7] R. Raghavan, K. Boopathy, R. Ghisleni, M.A. Pouchon, U. Ramamurty, J. Michler, Ion irradiation enhances the mechanical performance of metallic glasses, Scr. Mater. 62 (2010) 462-465.

[8] C. Dimitrov, H. Dammak, P. Legrand, A. Dunlop, D. Lesueur, Displacement damage and chemical disordering in short-range ordered alloys irradiated by swift heavy ions, Radiat. Effects Defects Solid 126 (1993) 115-118.

[9] M.T. Myers, S. Charnvanichborikarn, C.C. Wei, Z.P. Luo, G.Q. Xie, S.O. Kucheyev, D.A. Lucca, L. Shao, Phase transition, segregation and nanopore formation in high-energy heavy-ion-irradiated metallic glass, Scr. Mater. 67 (2012) $887-890$

[10] H. Zhang, X. Mei, Y. Wang, Z. Wang, Y. Wang, Resistance to $\mathrm{H}^{+}$induced irradiation damage in metallic glass $\mathrm{Fe}_{80} \mathrm{Si}_{7.43} \mathrm{~B}_{12.57}$, J. Nucl. Mater. 456 (2015) 344-350.
[11] K. Zhang, Z. Hu, F. Li, B. Wei, Viscous surface flow induced on Ti-based bulk metallic glass by heavy ion irradiation, Appl. Surf. Sci. 390 (2016) 941-945.

[12] S.G. Mayr, R.S. Averback, Effect of ion bombardment on stress in thin metal films, Phys. Rev. B 68 (2003) 214105

[13] M. Hou, S. Klaumünzer, G. Schumacher, Dimensional changes of metallic glasses during bombardment with fast heavy ions, Phys. Rev. B 41 (1990) 1144-1157.

[14] H. Trinkaus, A.I. Ryazanov, Viscoelastic model for the plastic flow of amorphous solids under energetic ion bombardment, Phys. Rev. Lett. 74 (1995) 5072-5075.

[15] H. Trinkaus, Local stress relaxation in thermal spikes as a possible cause for creep and macroscopic stress relaxation of amorphous solids under irradiation, J. Nucl. Mater. 223 (1995) 196-201.

[16] J.C. Ye, J. Lu, C.T. Liu, Q. Wang, Y. Yang, Atomistic free-volume zones and inelastic deformation of metallic glasses, Nat. Mater. 9 (2010) 619-623.

[17] X.L. Bian, G. Wang, H.C. Chen, L. Yan, J.G. Wang, Q. Wang, P.F. Hu, J.L. Ren, K.C. Chan, N. Zheng, A. Teresiak, Y.L. Gao, Q.J. Zhai, J. Eckert, J. Beadsworth, K.A. Dahmen, P.K. Liaw, Manipulation of free volumes in a metallic glass through Xe-ion irradiation, Acta Mater. 106 (2016) 66-77.

[18] Y. Petrusenko, A. Bakai, V. Borysenko, A. Astakhov, D. Barankov, Investigation of bulk metallic glass structure by means of electron irradiation, Intermetallics 17 (2009) 246-248.

[19] C.G. Zimmermann, M. Yeadon, K. Nordlund, J.M. Gibson, R.S. Averback, U. Herr, K. Samwer, Burrowing of Co nanoparticles on clean Cu and Ag surfaces, Phys. Rev. Lett. 83 (1999) 1163.

[20] R.M. Hengstler-Eger, P. Baldo, L. Beck, J. Dorner, K. Ertl, P.B. Hoffmann, C. Hugenschmidt, M.A. Kirk, W. Petry, P. Pikart, A. Rempel, Heavy ion irradiation induced dislocation loops in AREVA's M5 ${ }^{\circledR}$ alloy, J. Nucl. Mater. 423 (2012) $170-182$.

[21] Y. Liu, P. Zhang, Y.M. Zhang, J. Ding, J.J. Shi, Z.M. Sun, Spontaneous growth of Sn whiskers and a new formation mechanism, Mater. Lett. 178 (2016) 111-114.

[22] D. Niraula, J. McCulloch, G.R. Warrell, R. Irving, V.G. Karpov, D. Shvydka, Electric field stimulated growth of Zn whiskers, AIP Adv. 6 (2016) 075201

[23] F. Frost, R. Fechner, B. Ziberi, J. Vollner, D. Flamm, A. Schindler, Large area smoothing of surfaces by ion bombardment: fundamentals and applications, J. Phys.-Condens. Mater. 21 (2009) 224026.

[24] T. van Dillen, A. Polman, P.R. Onck, E. van der Giessen, Anisotropic plastic deformation by viscous flow in ion tracks, Phys. Rev. B 71 (2005) 024103

[25] C.A. Volkert, Density changes and viscous flow during structural relaxation of amorphous silicon, J. Appl. Phys. 74 (1993) 7107-7113. 